\title{
Hot hole relaxation in the SiGe system
}

\author{
K. Yeom, J. M. Hinckley, and J. Singh \\ Department of Electrical Engineering and Computer Science, The University of Michigan, Ann Arbor, \\ Michigan 48109-2122
}

(Received 4 May 1995; accepted for publication 21 July 1995)

\begin{abstract}
A general formalism is presented to study hot carrier relaxation in the valence band of strained and unstrained semiconductors. The approach is based on a six-band $k \cdot p$ description of the valence band and an anisotropic Monte Carlo method. We show the results of our studies for the $\mathrm{Si}-\mathrm{Ge}$ system. Carriers are initially injected in the split-off band and the carrier distribution is followed in time. Results are presented for energy-dependent energy relaxation time. The relaxation times for $\mathrm{Si}$ are about $10^{-13} \mathrm{~s}$ while those for Ge are an order of magnitude higher. To study the effect of biaxial strain produced through epitaxy on energy relaxation times we present results for $\mathrm{Si}_{0.8} \mathrm{Ge}_{0.2}$ on a $\{100\}$ silicon substrate. This work is of relevance to the interpretation of pump-probe experiments, which are primarily dependent on the relaxation of the average of the carrier energy. () 1996 American Institute of Physics. [S0021-8979(95)00122-9]
\end{abstract}

Hot carrier transport has been an important issue in both electronic and optoelectronic devices and energy relaxation is one of the most significant factors in hot carrier transport. Theoretical and experimental studies of hot carrier relaxation have primarily focussed on electrons in direct gap materials such as GaAs. ${ }^{1-6}$ These studies have been very valuable in understanding pump-probe studies using ultrafast lasers. It has been widely assumed that hot hole relaxation times are extremely fast and do not affect the interpretation of the pump-probe studies. With continuous improvements in the temporal resolution of the pump-probe studies, it is becoming possible to study phenomena at the time scales of $\sim 20$ fs-times which are relevant to hot hole relaxation. In this communication we present results on hot hole relaxation for the $\mathrm{Si}-\mathrm{Ge}$ system. Not only is this an important material system for technology, but it provides us a set of valenceband structures where the densities of states vary over almost an order of magnitude, especially near the band edges. The system also provides us an opportunity to study the effect of strain produced by epitaxial growth on carrier relaxation.

In modeling the valence-band structure, we use a sixband $k \cdot p$ Hamiltonian in which the effect of the strain can be included via the deformation potential theory. ${ }^{7,8}$ The effect of alloying is included through the virtual crystal approximation by a simple weighted average of the KohnLuttinger parameters. This band structure adequately describes the heavy-hole (HH), light-hole (LH), and split-off (SO) bands. When $\mathrm{Si}-\mathrm{Ge}$ is grown on a $\mathrm{Si}$ substrate [along the (001) direction] the strain removes the HH-LH degeneracy that occurs at $k=0$. This is known to reduce the nearband-edge density of states in the strained alloy. ${ }^{9}$ In this communication we focus on the following material systems:

(i) silicon;

(ii) unstrained $\mathrm{Si}_{0.8} \mathrm{Ge}_{0.2}$;

(iii) strained $\mathrm{Si}_{0.8} \mathrm{Ge}_{0.2}$ on a $\{100\}$ silicon substrate;

(iv) unstrained $\mathrm{Si}_{0.2} \mathrm{Ge}_{0.8}$; and

(v) germanium.

The SiGe system provides us sets of valence bands with the following interesting features. (i) The valence-band density of states in $\mathrm{Ge}$ is about an order of magnitude lower than that in $\mathrm{Si}$.

(ii) The strained alloy $\mathrm{Si}_{0.8} \mathrm{Ge}_{0.2}$ has a sharp reduction in the density of states near the band edge when compared with the unstrained alloy; however, as energy is above $0.1 \mathrm{eV}$, the density of the states becomes similar to that in the unstrained alloy.

(iii) Si has a relatively constant fractional density of states in the $\mathrm{HH}$ and $\mathrm{LH}$ bands as a function of the energy, while Ge does not.

The consequences of these effects are important to understand the hot hole relaxation in the SiGe system and are discussed below.

The Monte Carlo program used for this study includes the effect of band-structure anisotropies on the angular dependence of the scattering rates. The details of the Monte Carlo method are given in Refs. 10 and 11. Monte Carlo simulations are performed using acoustic- and opticalphonon scattering mechanisms. Alloy scattering is included as an additional scattering mechanism in the SiGe alloys. Among these scattering processes, optical-phonon emission and absorption play important roles in energy relaxation, because the other scattering processes are elastic. The holes in the calculation start with the same energy in the SO band. Results are reported for zero electric field and at $T=300 \mathrm{~K}$. During the calculation, average energy and band occupancy are recorded as a function of time.

In Fig. 1, the band occupancies in silicon and germanium are shown as a function of time. Carriers start in the SO band with an initial energy of $0.5 \mathrm{eV}$ for both material systems. Two observations must be made from the results shown:

(i) The carriers transfer out of the SO band very rapidly in both $\mathrm{Si}$ and $\mathrm{Ge}$;

(ii) for $\mathrm{Si}$ the ratio of the occupancy between the $\mathrm{HH}$ and LH bands reaches a constant value long before the carrier reaches equilibrium energy (this result is discussed in Fig. 2, below). 


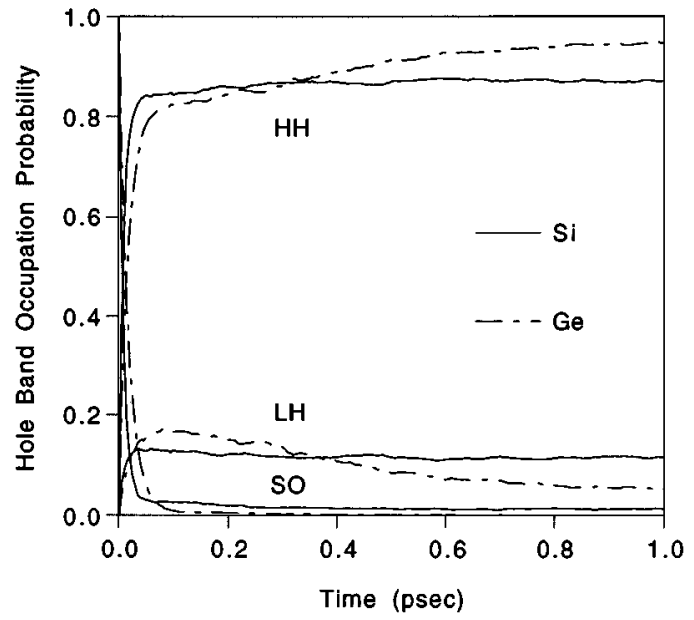

FIG. 1. Band occupancy of each band in silicon and germanium as a function of time. The results shown correspond to cases where holes with energy of $0.5 \mathrm{eV}$ are placed in the split-off band.

On the other hand for Ge, the $\mathrm{HH}$ and $\mathrm{LH}$ band occupation reaches a constant value only when the holes have reached their equilibrium energy.

To understand these observations we examine some of the scattering rates important for carrier relaxation and energy loss. We find that holes in the SO band of Si transfer to $\mathrm{HH}$ with an interband scattering rate that is 2.5 times larger than that in Ge, due to the high density of states in the silicon $\mathrm{HH}$ band. At $0.5 \mathrm{eV}$, the interband scattering rate from $\mathrm{SO}$ to $\mathrm{HH}$ in $\mathrm{Si}$ is approximately $1 \times 10^{14} \mathrm{~s}^{-1}$ and is an order of magnitude greater than the SO to LH scattering rate, and two orders of magnitude greater than the SO intraband scattering rate. Because of the high rates of interband transfer, holes are transferred to the $\mathrm{HH}$ band in approximately $50 \mathrm{fs}$ in $\mathrm{Si}$. In Ge this time is a little longer but it is still less than $100 \mathrm{fs}$.

To understand the second observation noted above, we examine the ratio of the density of states (DOS) of the $\mathrm{HH}$ and $\mathrm{LH}$ bands as a function of energy in Ge and Si. Figure 2 shows the ratio of DOS in each band to the total DOS. At

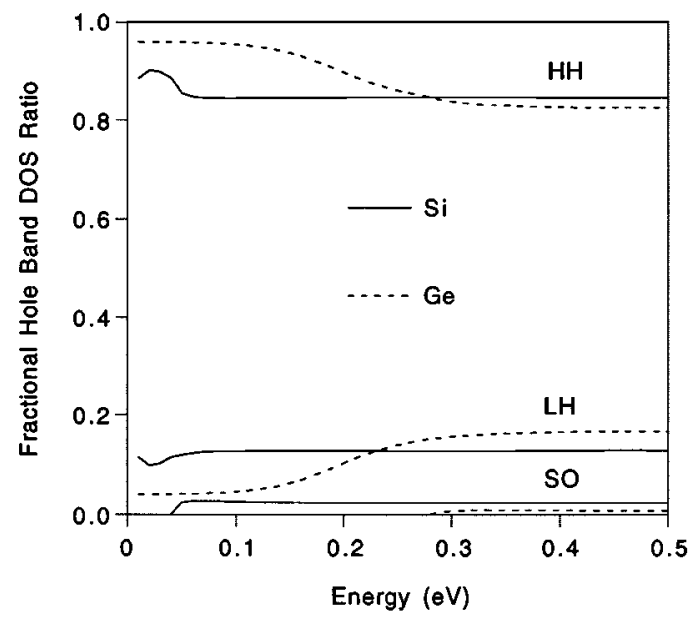

FIG. 2. Ratio of the density of states in each band to the total values in silicon and germanium as a function of energy.

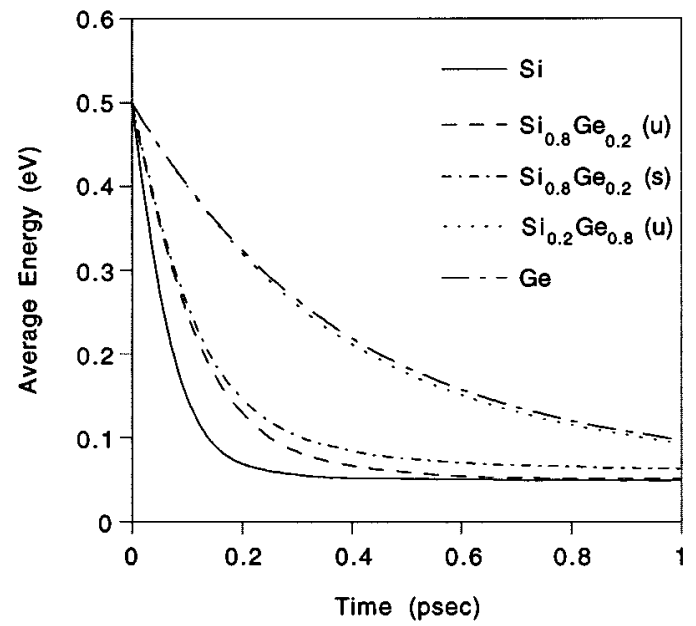

FIG. 3. Average energy in silicon, unstrained and strained $\mathrm{Si}_{0.8} \mathrm{Ge}_{0.2}$, unstrained $\mathrm{Si}_{0.2} \mathrm{Ge}_{0.8}$, and germanium. (u) and (s) represent unstrained and strained cases, respectively. In all cases, the holes are initially placed with $0.5 \mathrm{eV}$ energy in the split-off band.

high energies, Ge has roughly the same ratio of DOS in $\mathrm{HH}$ as in silicon but as we move closer to the band edge, while the $\mathrm{Si}$ ratio remains energy independent, in $\mathrm{Ge}$ it increases at $0.1 \mathrm{eV}$ and converges to a higher value than for $\mathrm{Si}$. Therefore, the band occupancy in Si reaches equilibrium value just after the transition from $\mathrm{SO}$ to $\mathrm{HH}$, while that in Ge takes a much longer time and comes to equilibrium only when the hole energy has thermalized.

In Fig. 3 the average energies in $\mathrm{Si}$, unstrained $\mathrm{Si}_{0.2} \mathrm{Ge}_{0.8}$, strained $\mathrm{Si}_{0.2} \mathrm{Ge}_{0.8}$, unstrained $\mathrm{Si}_{0.8} \mathrm{Ge}_{0.2}$, and $\mathrm{Ge}$ are presented as a function of time. The initial energy is $0.5 \mathrm{eV}$ for all cases. Due to the large scattering rates, holes in Si lose their energy much faster than those in Ge. The unstrained SiGe alloys have intermediate values between them, since energy relaxation is closely related to the DOS. The holes in the strained $\mathrm{Si}_{0.2} \mathrm{Ge}_{0.8}$ initially lose their energy with the same rate as for the unstrained system; however, they have larger relaxation time as their energy decreases. This is because the strained system has a lower effective mass at band edge due to the strain induced band deformation; therefore, the effect of the strain can be seen when the average energy is low. Results for other SiGe compositions can be inferred from the cases discussed in this communication since the relaxation times are closely related to the density of states of the alloy.

The results shown in Figs. 1 and 2 are for carriers initially injected at $0.5 \mathrm{eV}$. As the carriers relax, the energy distribution is no longer sharp but starts to spread around an average energy. To estimate the effect of this energy spread, we calculate an average energy dependent relaxation time. We define this relaxation time, $\tau$, through the temporal gradient of the average energy $\langle E\rangle$,

$$
\frac{1}{\tau(\langle E\rangle)}=-\frac{1}{\langle E\rangle} \frac{d\langle E\rangle}{d t} .
$$

In Fig. 4 the dependence of the energy-dependent relaxation time on the average energy is given in silicon and germa- 


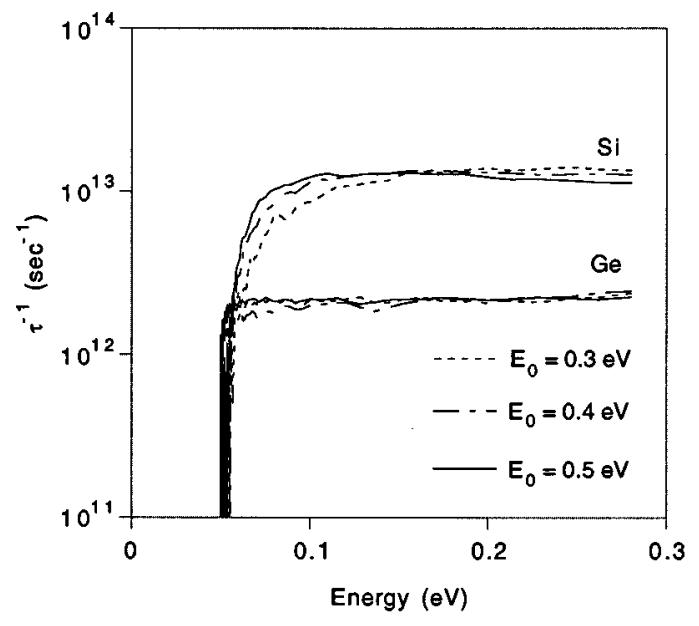

FIG. 4. Dependence of the energy relaxation time on the average energy in silicon and germanium. The relaxation time is defined by the temporal gradient of the average energy. The results are shown for three different initial energies.

nium. We use three initial conditions for these results. Holes are injected into the SO band with initial energies of $0.3,0.4$, and $0.5 \mathrm{eV}$. We see that even though the initial conditions are different in the three cases, the rate of energy relaxation is dependent on the average energy and is not significantly affected by the spread in the energy distribution.

In conclusion, energy relaxation in the valence band has been calculated using Monte Carlo simulations with a $k \cdot p$ band structure. We have shown results on carrier transfer times from the SO band to the $\mathrm{HH}$ and $\mathrm{LH}$ bands as well as the time evolution of the band occupancy. The effect of density of states and the influence of strain on carrier relaxation have also been discussed. We expect that the results presented here will be useful for understanding ultrafast laser pump-probe measurements focusing on hot hole relaxation. In such experiments it is the average carrier energy that is of importance, more than the details contained in the shape of the distribution function. Our results show that the intrinsic properties of the relaxation rates are such that its value at a given energy is virtually independent of the hole's initial energy.

This work was supported under the Army URI program (DAAL 03-92-G-0109) and under AFOSR Contract No. AFOSR 91-0349.

${ }^{1}$ S. D. Sarma, V. B. Campos, M. A. Stroscio, and K. W. Kim, Semicond. Sci. Technol. 7, B60 (1992).

${ }^{2}$ M. A. Cavicchia and R. R. Alfano, Phys. Rev. B 48, 5696 (1993).

${ }^{3}$ A. Straw, A. J. Vickers, and J. S. Roberts, Semicond. Sci. Technol. 7, B343 (1992)

${ }^{4}$ J. Lutz, F. Kuchar, K. Ismail, H. Nickel, and W. Schlapp, Semicond. Sci. Technol. 8, 399 (1993).

${ }^{5}$ T. Furuta, N. Shigekawa, and M. Tomizawa, Semicond. Sci. Technol. 9, 453 (1994).

${ }^{6}$ N. G. Asmar, A. G. Markelz, E. G. Gwinn, P. F. Hopkins, and A. C. Gossard, Semicond. Sci. Technol. 9, 828 (1994).

${ }^{7}$ G. Dresselhaus, A. F. Kip, and C. Kittel, Phys. Rev. 98, 368 (1955).

${ }^{8}$ J. M. Luttinger, Phys. Rev. 102, 1030 (1956).

${ }^{9} \mathrm{~J}$. Singh, Physics of Semiconductors and Their Heterojunctions (McGraw-Hill, New York, 1993), pp. 238-245.

${ }^{10}$ J. M. Hinckley and J. Singh, Phys. Rev. B 42, 3546 (1990).

${ }^{11}$ J. M. Hinckley and J. Singh, J. Appl. Phys. 76, 4192 (1994) 